\title{
EL ACCESO A LOS MEDICAMENTOS: CONFLICTOS ENTRE DERECHOS DE PROPIEDAD INTELECTUAL Y PROTECCIÓN DE LA SALUD PÚBLICA
}

\begin{abstract}
Raúl Allard Soto ${ }^{1}$
Resumen: Entre los mayores obstáculos que enfrenta en todo el mundo el acceso a los medicamentos se encuentran las cuestiones relativas a los derechos de propiedad intelectual y la falta de competencia, unido al retardo y postergación en la generación de investigación y desarrollo (I+D) sobre aquellas enfermedades que afectan de manera importante a la población de países en desarrollo y países menos adelantados. En este trabajo se analiza el fortalecimiento de la patente farmacéutica contemplada en el Acuerdo sobre los Aspectos de los Derechos de Propiedad Intelectual (ADPIC) de la Organización Mundial de Comercio $(\mathrm{OMC})$ y el actual régimen de incentivo imperante para medicamentos nuevos, sus consecuencias y actuales conflictos. En ellos suelen enfrentarse los intereses de países desarrollados, presionados por una poderosa industria farmacéutica, con los intereses de países en desarrollo y países menos adelantados.
\end{abstract}

Palabras clave: Acuerdo ADPIC, patente farmacéutica, acceso a medicamentos, I+D

\section{The access to drugs: conflicts between intellectual property and public health protection}

\begin{abstract}
Topics related to intellectual property rights and lack of competency are found among the major obstacles facing the access to drugs in the world, together with the delay and postponement in the generation of R\&D about those diseases affecting disproportionately to the population of developing countries and least developed countries. In this study, the strengthening of pharmaceutical patents included in the Agreement on trade-related aspects of intellectual property rights of the World Trade Organization (WTO) and the current prevailing incentive towards new drugs, their consequences and current conflicts are analyzed. In these, generally, the developed countries interests are confronted -pressed by a powerful pharmaceutical industry- with the interests of developing countries and least developed countries.
\end{abstract}

Key words: Agreement on trade-related aspects of intellectual property rights, pharmaceutical patent, access to drugs, R\&D

O acesso aos medicamentos: conflitos entre direitos de propriedade intelectual e proteçáo da saúde pública

Resumo: Entre os maiores obstáculos que enfrenta em todo o mundo, o acesso aos medicamentos se encontram as questôes relativas aos direitos de propriedade intelectual e a falta de competência, aliado ao retardo e postergaçáo na geração de $\mathrm{I}+\mathrm{D}$ sobre aquelas enfermidades que afetam de maneira desproporcionada a população dos países em desenvolvimento e países menos adiantados. Neste trabalho se analisa o fortalecimento da patente farmacêutica contemplada no Acordo sobre os Aspectos dos Direitos de Propriedade Intelectual (ADPIC) da Organização Mundial de Comércio (OMC) e o atual regime de incentivo imperante para medicamentos novos, suas consequências e atuais conflitos. Neles devem ser enfrentados os interesses de países desenvolvidos - pressionados por uma poderosa indústria farmacêutica - com os interesses de países em desenvolvimento e países menos adiantados.

Palavras-chave: Acordo ADPIC, patente farmacêutica, acesso a medicamentos, I+D

\footnotetext{
${ }^{1}$ Departamento de Ciencias Jurídicas, Facultad de Ciencias Jurídicas y Empresariales, Universidad de la Frontera, Temuco, Chile Correspondencia: raul.allard@ufrontera.cl
} 


\section{Introducción}

La ampliación y el reforzamiento de los derechos de los titulares de patentes farmacéuticas, dispuesto en el Acuerdo sobre los Aspectos de los Derechos de Propiedad Intelectual relacionados con el Comercio (en adelante "Acuerdo ADPIC") ${ }^{2}$, de 1995, de la Organización Mundial del Comercio (OMC), tensionan mundialmente el acceso a los medicamentos.

Estas patentes confieren a su titular - habitualmente empresas farmacéuticas globales, con casa matriz en Estados Unidos y Europa- derechos exclusivos de explotación ${ }^{3}$ por un periodo mínimo de 20 años sobre el nuevo medicamento, que retrasan la entrada al mercado del medicamento genérico $^{4}$. En definitiva, el Acuerdo ADPIC fija un estándar mínimo mundial para la protección de monopolios de propiedad intelectual de dominio privado, vinculante para todos los Estados miembros de la OMC. Este periodo de exclusión permite al titular recuperar parte de los costos involucrados en la investigación médica.

Por ello, al abordar los nuevos problemas que enfrenta en la actualidad el acceso a los medicamentos, la Relatoría Especial de las Naciones Unidas sobre el derecho de toda persona al disfrute del más alto nivel posible de salud física y mental (en adelante "Relatoría Especial"), identifica como aquellos obstáculos de mayor envergadura las cuestiones relativas a los derechos de propiedad intelectual y la falta de competencia. Además, considera que la competencia es el elemento primordial para reducir los precios de los medicamentos y lograr su asequibilidad para todos, subrayando que la única competencia eficaz es la creada por los medicamentos genéricos(1).

\footnotetext{
${ }^{2}$ El Acuerdo ADPIC es un acuerdo anexo a la creación de la OMC, el cual requiere ser suscrito por los países que deseen ser miembros de la OMC. Es quizás su acuerdo más controvertido, porque países desarrollados presionaron a favor de una amplia protección de la propiedad intelectual y la armonización de las normas al respecto. Publicado en el Diario Oficial, República de Chile, 17 de mayo de 1995.

${ }^{3}$ Artículo 28, Acuerdo ADPIC.

${ }^{4}$ Aquellos que no están amparados por una patente farmacéutica, que se comercializan bajo el nombre del principio activo y cuya competencia usualmente conduce a una reducción significativa de los precios y a un mayor acceso.
}

Por tanto, los efectos eventualmente negativos que pueden llegar a provocar los acuerdos de la OMC en el derecho a la salud(2), así como las diversas controversias suscitadas entre sus Estados miembros —específicamente en el ámbito de las patentes farmacéuticas - con motivo de la interpretación y aplicación de las disposiciones del Acuerdo ADPIC(3), atestiguan esta tensión. En ellos suelen confrontarse los derechos de propiedad intelectual con la adopción de medidas en protección de la salud pública.

\section{Acceso a medicamentos y leyes de propiedad inte- lectual}

El acceso a los medicamentos presenta cuatro aspectos: deben ser accesibles en todo el país; ser económicamente asequibles para todos, incluidas las personas que viven en la pobreza; ser accesibles sin discriminación por ninguno de los motivos prohibidos y, por último, los pacientes y profesionales de la salud deben tener acceso a información fidedigna sobre ellos, para poder adoptar decisiones con conocimiento de causa. De ellos, la Relatoría Especial, al examinar la relación entre las leyes de propiedad intelectual y el acceso a los medicamentos, atribuye especial relevancia al aspecto de la accesibilidad económica, ante la incidencia significativa que dichas regulaciones suelen provocar nada menos que en el incremento del precio del fármaco. Por tal motivo, los Estados, en cumplimiento de las obligaciones contraídas en el marco del derecho internacional, deben necesariamente asegurar que sus regímenes nacionales de propiedad intelectual protejan el derecho a la $\operatorname{salud}(4)$.

Pero, al mismo tiempo, junto a la accesibilidad económica de los medicamentos actualmente existentes, también resulta fundamental, para asegurar el derecho al nivel de salud más alto posible, que los medicamentos nuevos que sean muy necesarios se produzcan lo antes posible. De hecho, el retardo en la generación de investigación y desarrollo $(\mathrm{I}+\mathrm{D})$ que sufren determinadas enfermedades ha dado lugar a las "enfermedades desatendidas" (5-8), aquellas que no resultan rentables económicamente por no ser capaces de generar una demanda de medicamentos y un mercado en condiciones económicas de adquirirlos, afectando de manera desproporcionada a la población de los 
países en desarrollo y países menos adelantados 5 . Aquí también se produce una intersección crucial entre derechos de propiedad intelectual y la generación de $\mathrm{I}+\mathrm{D}$, otro de los factores determinantes del acceso a los medicamentos $(6,7)$.

De tal manera, este trabajo aborda aquellos conflictos principales que afectan el acceso a los medicamentos a nivel mundial. En ellos, las tensiones entre los derechos de propiedad intelectual y la protección de la salud pública afloran protagónicamente.

\section{Declaración de Doha}

$\mathrm{Al}$ establecer normas mundiales mínimas sobre los principales derechos de propiedad intelectual, la aplicación del Acuerdo ADPIC implicó para la mayoría de los países en desarrollo y países menos adelantados la actualización de sus normas de propiedad intelectual, mediante un conjunto complejo de reformas para volver a redactar y actualizar la legislación vigente(9). Como grafica Sell, el Acuerdo ADPIC refleja el interés de empresas globales que intentan ampliar su control sobre la propiedad intelectual: "amplía los derechos de patente hasta los 20 años; obliga a los países en desarrollo a otorgar protección mediante patente a los productos farmacéuticos; restringe de manera estricta las condiciones en que los Estados pueden expedir licencias obligatorias ${ }^{6}$, y reduce la autonomía de los Estados en el diseño de políticas de propiedad intelectual que se ajusten a sus diversos niveles de innovación y desarrollo económico"(10:172-173).

De tal manera, muy pronto —el año 2001, seis años después de su adopción- los efectos negativos en el acceso a los medicamentos que generaba el Acuerdo ADPIC se hacen sentir en los países en desarrollo y países menos adelantados, obligando a los Estados miembros de la OMC a emitir la Declaración de Doha relativa al Acuerdo

\footnotetext{
${ }^{5}$ En ocasiones se denominan enfermedades tropicales o relacionadas con la pobreza, e incluyen, por ejemplo, la leishmaniasis, la oncocercosis, la enfermedad de Chagas, la lepra, la esquistosomiasis, la filariasis linfática, la tripanosomiasis africana y el dengue. Aunque en ańos recientes el VIH/SIDA, la tuberculosis y la malaria han recibido una atención y recursos crecientes, también pueden considerarse enfermedades desatendidas(5:17).

${ }^{6}$ Por "licencia obligatoria" se entiende el permiso que da un gobierno para producir un producto patentado o utilizar un procedimiento patentado sin el consentimiento del titular de la patente.
}

sobre los ADPIC y la Salud Pública ("Declaración de Doha”), cuyo principal mérito estriba en el reconocimiento explícito de esta situación: en particular, en nuestro concepto, el reconocimiento de las preocupaciones respecto de los efectos de la protección de la propiedad intelectual sobre los precios de los medicamentos ${ }^{7}$ y el reconocimiento de que las "flexibilidades ADPIC"8 incluyen el derecho de cada Estado miembro de conceder licencias obligatorias y la libertad de determinar las bases sobre las cuales se conceden tales licencias?. Junto a ello, los Estados miembros convienen que el Acuerdo ADPIC no puede impedir que los miembros adopten medidas para proteger la salud pública y, en particular, promuevan el acceso a los medicamentos para todos ${ }^{10}$.

Por ello, concordamos con Sell cuando afirma que "la batalla sobre el acceso a los medicamentos básicos gira en torno a los derechos de expedición de licencias obligatorias y a la producción y exportación de versiones genéricas de medicamentos de marca, con el objetivo de ampliar el acceso"(10:175). Controversias emblemáticas vertidas por los Estados miembros ante el Órgano de Solución de Diferencias (OSD) comerciales de la OMC, sobre patentes farmacéuticas, confirman aquella disputa, que la mayoría de las veces enfrenta los intereses de países desarrollados - influenciados por una poderosa industria farmacéutica- con los intereses de países en desarrollo.

\section{Controversias sobre patentes farmacéuticas ante el OSD}

El sistema de solución de diferencias comerciales se compone de diversas etapas(11): primero, la solicitud de celebración de consultas, cuando un país reclama contra la conducta de otro en la

\footnotetext{
7 Declaración de Doha relativa al Acuerdo sobre los ADPIC y la Salud Pública, segunda parte, párrafo 3.

${ }^{8}$ Disposiciones del Acuerdo ADPIC que habilitan la aplicación de medidas que permiten templar los derechos de patente y proteger la salud pública, tales como: concesión de licencias obligatorias; calificación de casos de emergencias nacionales y otras circunstancias de extrema urgencia (por ejemplo, epidemias); excepciones con fines de investigación y experimentación; excepción basada en el examen reglamentario o "cláusula bolar"; importaciones paralelas y agotamiento de derechos.

9 Declaración de Doha relativa al Acuerdo sobre los ADPIC y la Salud Pública, párrafo 5, letra b.

${ }^{10}$ Declaración de Doha relativa al Acuerdo sobre los ADPIC y la Salud Pública, párrafo 4.
} 
ejecución y aplicación de un acuerdo de la OMC y solicita "sentarse a conversar" con él; segundo, de no alcanzar una negociación sobre la diferencia, el reclamante pide la "constitución de Grupo Especial" — panel de expertos- que elabora un informe resolviendo la diferencia, haciendo las veces de tribunal de primera instancia. Tercero, de existir disconformidad de una parte o de ambas por la resolución del Grupo Especial, pasa al Órgano de Apelación, cuya resolución final resuelve el litigio y recomienda al perdedor la aplicación de determinadas medidas con el fin de dejar de transgredir el acuerdo de la OMC; finalmente, si esta recomendación no se cumple en un plazo determinado, el reclamante puede solicitar la aplicación de sanciones comerciales al perdedor o "retorsiones".

Hasta el momento, los Estados miembros de la OMC han presentado nueve controversias ante el OSD con motivo de la interpretación y aplicación de las disposiciones del Acuerdo ADPIC sobre patentes farmacéuticas(3:239-270). La relevancia de este procedimiento se manifiesta en su carácter vinculante y obligatorio(12). Varios de ellos implicaron en definitiva la adecuación y modificación de la legislación interna del Estado miembro de la OMC a las normas del Acuerdo ADPIC ${ }^{11}$.

Asimismo, en las controversias suele graficarse el enfrentamiento entre derechos de propiedad intelectual promovidos en defensa de empresas farmacéuticas, por una parte, y los requerimientos de protección de la salud pública, por otra. A continuación aludiremos a algunas de ellas, por involucrar aspectos, en nuestro concepto cruciales, del acceso a los medicamentos.

\section{Producción y exportación de genéricos}

En la actualidad, dos controversias emblemáticas

\footnotetext{
${ }^{11}$ Estados Unidos contra India (1996): Diferencia de Solución (D.S.) No 50, caratulada "India - Protección mediante patente de los productos farmacéuticos y los productos químicos para la agricultura"; Comunidades Europeas contra India (1997): D.S. No79, caratulada "India - Protección mediante patente de los productos farmacéuticos y los productos químicos para la agricultura”; Comunidades Europeas contra Canadá (1997): D.S. No114, caratulada "Canadá - Protección mediante patente de los productos farmacéuticos”; y Estados Unidos contra Argentina (2000): D.S. No196, caratulada "Argentina - Determinadas medidas relativas a la protección de patentes y de los datos de pruebas".
}

para el futuro del suministro de medicamentos genéricos se encuentran pendientes de avance y resolución ante el OSD desde 2010: la primera enfrenta en calidad de reclamante a la India el proveedor principal de medicamentos genéricos para los países en desarrollo y países menos adelantados- contra la Unión Europea ${ }^{12}$, y la segunda confronta a Brasil en calidad de reclamante contra la Unión Europea ${ }^{13}$.

Ambas, íntimamente vinculadas entre sí, se presentaron en mayo del 2010 ante el OSD por reiteradas confiscaciones a exportaciones de medicamentos genéricos — clopidogrel, abacavir, olanzapina, rivastigmina y losartán, entre otros-, originarios mayoritariamente desde la India, en tránsito por puertos y aeropuertos de los Países Bajos, con destino hacia países en desarrollo y países menos adelantados. Las confiscaciones han permitido a grandes empresas farmacéuticas - Novartis, Glaxo, E.I. Dupont de Nemours, Sanofi-Aventis, Eli Lilly, Merck, entre otras- valerse de las severas leyes de patentes del bloque europeo para hacer que sus aduanas nacionales embarguen reiteradamente medicamentos genéricos en tránsito, bajo el argumento de que violan leyes de patentes de la Unión Europea. Muchos remedios genéricos indios contra el tratamiento del VIH/SIDA sufrieron esta suerte.

Según reclaman la India y Brasil, medicamentos genéricos fabricados en la India y en tránsito a terceros países —entre ellos Colombia, Nigeria, Perú y Brasil- fueron tratados como si se hubieran fabricado realmente en los Países Bajos, siendo retenidos inicialmente y más tarde destruidos o devueltos a la India y, solo en unos pocos casos, se permitió que los envíos siguieran su camino hasta el país de destino después de considerables demoras.

Para ambos países dichas acciones contravienen

\footnotetext{
${ }^{12}$ India contra Unión Europea (2010): D.S. No 408, caratulada "Unión Europea y un Estado Miembro - Confiscación de medicamentos genéricos en tránsito". Para revisar historia completa del caso, véase sitio www.wto.org/spanish/tratop s/dispu s/cases s/ ds408_s.htm (visitado el 26 de septiembre de 2013).

${ }^{13}$ Brasil contra Unión Europea (2010): D.S. No409, caratulada "Unión Europea y un Estado Miembro - Confiscación de medicamentos genéricos en tránsito". Para revisar historia completa del caso, véase sitio www.wto.org/spanish/tratop_s/dispu_s/cases_s/ ds409 s.htm (visitado el 26 de septiembre de 2013).
} 
diversos artículos del Acuerdo ADPIC ${ }^{14}$. La India pone el énfasis en que dichas medidas crean obstáculos al comercio legítimo, constituyen un claro caso de abuso de derechos de patente y permiten obstaculizar la libertad de tránsito de medicamentos que se pueden producir y exportar desde la India a miembros de la OMC con capacidad insuficiente o inexistente en el sector farmacéutico, que tratan de obtener suministros de esos productos para hacer frente a sus problemas de salud pública, haciendo un uso efectivo de las licencias obligatorias. Brasil pone el énfasis en que la aplicación del Reglamento No 1383/2003 del Consejo de las Comunidades Europeas, en el que se basaron las autoridades neerlandesas para confiscar la expedición del medicamento genérico potasio de losartán, producido en la India y con destino a Brasil — con independencia de la situación de la patente del medicamento en los países de origen y destino-, es incompatible con las obligaciones que corresponden a la Unión Europea y a los Países Bajos en virtud del Acuerdo de Marrakech, por el que se establece la propia OMC y los Acuerdos anexos al mismo, incluido el Acuerdo ADPIC.

El próximo paso en este procedimiento debiera ser la constitución de un Grupo Especial, toda vez que no se ha notificado la adopción de una solución mutuamente convenida. Como se puede ver, temas altamente sensibles para el acceso a medicamentos - tratados en sede comercial — no se encuentran aclarados ni resueltos a favor de la protección de la salud pública. Ni más ni menos que el que la India, con una industria farmacéutica de tradición mundial, pueda continuar produciendo, suministrando y exportando genéricos a países en desarrollo, como queda de manifiesto en estas dos controversias. Además, unido a ello, la dificultad que añade el Acuerdo ADPIC $^{15}$ para la importación de medicamentos genéricos por parte de países que tienen limitada o nula capacidad de fabricación de productos farmacéuticos, al limitar sus posibilidades de concesión de licencias

\footnotetext{
${ }^{14}$ Artículos 2, 28, 31, 41 y 42, Acuerdo ADPIC.

${ }^{15}$ Artículo 31 Acuerdo ADPIC: "Otros usos sin autorización del titular de los derechos. Cuando la legislación de un Miembro permita otros usos de la materia de una patente sin autorización del titular de los derechos, incluido el uso por el gobierno o por terceros autorizados por el gobierno, se observarán las siguientes disposiciones: (...) f) se autorizarán esos usos principalmente para abastecer el mercado interno del Miembro que autorice tales usos".
}

obligatorias para abastecer su mercado interno, como lo reconoce la Declaración de Doha ${ }^{16}$.

\section{Expedición de licencias obligatorias}

El artículo 31 del Acuerdo ADPIC permite a los miembros de la OMC expedir leyes nacionales que permitan el uso de una materia objeto de la patente sin la autorización del titular de los derechos sobre la misma, si se cumplen ciertos requisitos(13). Por su ambigüedad, aquellos requisitos dificultan su implementación $(14,15)$ y, si bien muchos países han adoptado mecanismos en sus legislaciones nacionales para conceder licencias obligatorias, los motivos son variados - negativa del titular de la patente a conceder licencias, interés público, salud pública y nutrición de la población, emergencia nacional u otras circunstancias de extrema urgencia, prácticas anticompetitivas, patentes dependientes y falta o insuficiencia de explotación- y los procedimientos previstos para su aplicación no son uniformes. Con todo, la incorporación de esta flexibilidad ADPIC en las legislaciones nacionales y su posterior uso por los respectivos gobiernos es fuertemente resistida por las empresas farmacéuticas.

De tal manera, la expedición de licencias obligatorias constituye otro aspecto crucial en el que se produce una colisión frontal entre los derechos de propiedad intelectual y las necesidades de protección de la salud pública, sobre todo en situaciones de emergencia nacional causadas por crisis de salud pública(15:168). A continuación se alude a una controversia que ilustra las dificultades que enfrentan los países en desarrollo para dar curso a una licencia obligatoria, más aún si tenemos en cuenta que quien enfrenta dichas dificultades es ni más ni menos que el gigante sudamericano Brasil, en plena crisis de VIH/SIDA. El reclamante es Estados Unidos.

Esta controversia $^{17}$ se origina por la incorporación

${ }^{16}$ Declaración de Doha, párrafo 6, primera parte: "Reconocemos que los Miembros de la OMC cuyas capacidades de fabricación en el sector farmacéutico son insuficientes o inexistentes podrían tropezar con dificultades para hacer un uso efectivo de las licencias obligatorias con arreglo al Acuerdo sobre los ADPIC".

${ }^{17}$ Estados Unidos contra Brasil (2000): D.S. No199, caratulada "Brasil - Medidas que afectan la protección mediante patente". Para revisar historia completa del caso, véase sitio www.wto.org/spanish/ tratop_s/dispu_s/cases_s/ds199_s.htm (visitado el 26 de septiembre de 2013). 
en la Ley de Propiedad Industrial de 1996 de Brasil de un requisito de explotación "local" —artículo 68- para el disfrute de derechos exclusivos de patente, que solo podrá cumplirse mediante la producción nacional y no la importación de la materia patentada, de lo contrario puede ser objeto de una licencia obligatoria si no se "explota" en el territorio de Brasil. Ello para impedir el incremento del precio que genera la importación del medicamento por quien detenta la titularidad de la patente. Para Estados Unidos ${ }^{18}$ esta disposición era incompatible con las obligaciones relativas a los derechos de patente contraídas por Brasil en el Acuerdo ADPIC.

En definitiva, los gobiernos de ambos países notificaron al OSD haber acordado una solución mutuamente convenida ${ }^{19}$. Ella consistía en que el gobierno del Brasil, en caso de considerar necesario conceder una licencia obligatoria respecto de las patentes de empresas de Estados Unidos, aceptaba celebrar conversaciones previas sobre el asunto con el gobierno de Estados Unidos para debatir el tema. En todo caso, en el propio texto del acuerdo, Estados Unidos dejaba constancia que Brasil no había usado nunca esta disposición para conceder una licencia obligatoria, lo que resulta muy revelador para nuestro tema de análisis.

\section{Excepción basada en el examen reglamentario}

La excepción "basada en el examen reglamentario" o cláusula "Bolar" constituye, a nuestro juicio, otra arista fundamental para el acceso a medicamentos. Permite que un tercero, no titular de la patente, importe, fabrique y utilice un producto patentado antes de su expiración, con el solo objeto de obtener la autorización de comercialización ante la autoridad sanitaria respectiva. De esta manera, se acelera el proceso de registro sanitario y se facilita la entrada del medicamento genérico en el mercado, para que pueda estar disponible a la venta tan pronto expire la patente farmacéutica.

El cumplimiento de los requisitos de registro sanitario antes del vencimiento de la protección

\footnotetext{
${ }^{18}$ Solicitud de celebración de consultas presentada por los Estados Unidos el 8 de junio de 2000, (WT/DS199/1).

${ }^{19}$ Solución mutuamente convenida, de 19 de julio de 2001, (WT/ DS199/4).
}

de la patente farmacéutica facilita la entrada del medicamento genérico en el mercado al día siguiente de su expiración(4:17). De este modo, se evita que, en la práctica, el titular de una patente farmacéutica goce de un periodo de exclusividad incluso mayor a los 20 años de protección fijado como estándar mínimo en el Acuerdo ADPIC.

La excepción reglamentaria se consagró como una importante flexibilidad ADPIC en la controversia que enfrentó a las Comunidades Europeas contra Canadá ${ }^{20}$. La reclamación se presentó en 1997, por la incorporación de dos disposiciones en la Ley de Patentes de Canadá: una relativa al examen reglamentario (artículo 55.2.1) y otra al almacenamiento (artículo 55.2.2). Aquellas disposiciones, en opinión del reclamante "Comunidades Europeas", vulneran los derechos exclusivos de patente consagrados en el Acuerdo ADPIC, por cuanto la primera permite a los fabricantes generales de productos farmacéuticos producir muestras del producto patentado para su uso durante el proceso de examen reglamentario ante las autoridades de salud, y la segunda permite a los productores de medicamentos genéricos fabricar esos medicamentos y empezar a acumularlos seis meses antes de la expiración de la patente. Para Canadá, ambas disposiciones se encontraban amparadas y cubiertas por el Acuerdo ADPIC, que permite a los países miembros prever excepciones limitadas ${ }^{21}$ de los derechos exclusivos conferidos por una patente, teniendo en cuenta que la comercialización solamente se efectuará expirada la patente.

En definitiva, a falta de solución amistosa, el Informe del Grupo Especial del OSD concluyó que la disposición relativa al examen reglamentario era compatible con las obligaciones que impone a Canadá el Acuerdo sobre los ADPIC ${ }^{22}$. En

\footnotetext{
${ }^{20}$ Comunidades Europeas contra Canadá (1997): D.S. No114, caratulada "Canadá - Protección mediante patente de los productos farmacéuticos”. Para revisar historia completa del caso, véase sitio www.wto.org/spanish/tratop_s/dispu_s/cases_s/ds114_s.htm (visitado el 26 de septiembre de 2013).

${ }^{21}$ Para que una medida excepcional se encuentre justificada, al amparo del artículo 30 del Acuerdo ADPIC, deben satisfacerse tres criterios acumulativos: i) tiene que ser limitada; ii) no debe "atentar de manera injustificable contra la explotación normal de la patente"; y iii) no debe "causar un perjuicio injustificado a los legítimos intereses del titular de la patente", teniendo en cuenta los intereses legítimos de terceros.

${ }^{22}$ Informe del Grupo Especial, adoptado el 17 de marzo de 2000,
} 
cambio, la disposición relativa al almacenamiento fue considerada incompatible con el Acuerdo $\mathrm{ADPIC}^{23}$. De tal manera, esta controversia debe ser considerada la de mayor relevancia que ha resuelto hasta el momento el OSD en beneficio del acceso a los medicamentos.

Ahora bien, lamentablemente muchos países en desarrollo y países menos adelantados, al celebrar Tratados de Libre Comercio (TLC) de carácter bilateral o multilateral con países desarrollados, incorporan entre sus disposiciones normas "ADPIC Plus", que refuerzan la protección de las patentes farmacéuticas con mayor vigor que el propio Acuerdo ADPIC. Uno de los objetivos de estas normas "ADPIC Plus" suele ser introducir la vinculación entre las patentes, el registro y la aprobación de los medicamentos(4:25-30).

Un ejemplo de ello es el TLC Chile-Estados Unidos(16), que permite negar el registro de un medicamento ante la autoridad sanitaria - aun cuando el solicitante del registro sanitario cumpla plenamente con los requisitos para su aprobación- de comprobarse la existencia de una patente farmacéutica, unido a una serie de obligaciones para con el titular de la patente farmacéuti$\mathrm{Ca}^{24}$. Para dar cumplimiento al TLC, el gobierno de Chile presentó en la Cámara de Diputados, a principios de 2012, un proyecto de ley ${ }^{25}$ que regula el "linkage", figura que, en oposición a la excepción basada en el examen reglamentario, alude a la no concesión de registros sanitarios por el Instituto de Salud Pública de Chile, atendida la existencia de patentes vigentes para el producto farmacéutico ante el Instituto Nacional de Propiedad Industrial. De aprobarse, puede provocar un retardo aún mayor en la entrada de medicamentos genéricos al mercado.

\section{I+D de medicamentos nuevos}

Por su parte, la protección de la propiedad intelectual y el régimen jurídico que se adopta puede afectar la investigación médica — si incide finalmente en la definición de medicamentos nuevos

\footnotetext{
(WT/DS114/R), párrafo 7.84.

${ }^{23}$ Informe del Grupo Especial, párrafo 7.33.

${ }^{24}$ Tratado de Libre Comercio Chile-Estados Unidos: artículos 17.9.4 y 17.10.2.

${ }^{25}$ No Boletín: 8183-03.
}

que se prioricen para generar $\mathrm{I}+\mathrm{D}-\mathrm{y}$ esto provocar efectos relevantes en el acceso a medicamentos. Es decir, también en la generación de $\mathrm{I}+\mathrm{D}$ para nuevos medicamentos irrumpen tensiones evidentes entre el régimen mundial imperante de derechos de propiedad intelectual y la protección de la salud pública: ¿ $\mathrm{I}+\mathrm{D}$ basada en necesidades de mercado o I+D basada en necesidades sanitarias de la población?

Insertos en el actual régimen de incentivos de derechos de propiedad intelectual que hemos estado analizando, aproximadamente el $90 \%$ de la $\mathrm{I}+\mathrm{D}$ de medicamentos es financiada por la industria farmacéutica(17) y ello puede comprometer la integridad de la ciencia $(18,19)$. La protección mediante una patente puede promover investigación médica al ayudar a la industria farmacéutica a solventar los costos de probar, desarrollar y aprobar medicamentos: esa es la idea fuerza detrás del actual régimen de propiedad intelectual. Ahora bien, como advierte Páez, "el riesgo es quedar a merced de los dictados propios del mercado, los cuales pueden interferir con los fines de la investigación científica, uno de los cuales es crear conocimiento para remediar las grandes necesidades en salud", de manera que "el descubrimiento y el desarrollo de nuevos medicamentos por la industria farmacéutica están obligados a servir no solo a intereses científicos o médicos, sino también a la eficiencia económica" (20:238).

\section{Desequilibrio 90/10}

Ya en 1990 la Comisión de Investigaciones Sanitarias para el Desarrollo señaló lo que llegó a conocerse como el desequilibrio 90/10: solo un $10 \%$ de los gastos de $\mathrm{I}+\mathrm{D}$ se dirige a los problemas de salud de un $90 \%$ de la población mundial. Es interesante el hecho de que el informe original cuantificara en realidad el desequilibrio en $95 / 5$, una proporción probablemente más realista. Este informe condujo a la creación en 1996 del Global Forum for Health Research, que sigue documentando la profunda ineficiencia del esfuerzo investigador orientado a las enfermedades de los pobres(7:87).

Vale explicar esta desigualdad recurriendo a la clasificación de enfermedades de la Comisión de Macroeconomía y Salud de la OMS, cuya tipo- 
logía se basa precisamente en la generación de I+D y el acceso a estas tecnologías (7:83-94): en las enfermedades de tipo $I$ - que afectan tanto a los países ricos como a los países pobres, con una amplia población vulnerable en unos y otrosexisten incentivos para la $\mathrm{I}+\mathrm{D}$ en los mercados de los países ricos, en cambio en los países pobres, el principal problema de política es el acceso a estas tecnologías, generalmente de alto precio y protegidas por patentes; en las de tipo II — que afectan tanto a los países ricos como a los países pobres, con la salvedad que una proporción importante de los casos se localizan en estos últimos-, existen incentivos para la $\mathrm{I}+\mathrm{D}$ en los mercados de los países ricos, pero el nivel de gasto en $\mathrm{I}+\mathrm{D}$ a escala mundial no se corresponde con la carga de morbilidad; y en las de tipo III — que afectan de manera muy predominante o exclusiva a los países en desarrollo-, estas enfermedades son objeto de una escasísima I+D que, en su vertiente comercial, es casi nula en los países ricos. De hecho, las enfermedades de tipo II se denominan a menudo enfermedades desatendidas y las de tipo III enfermedades muy desatendidas.

Las siguientes estadísticas ilustran bien el conflicto. De 1.393 nuevos medicamentos aprobados entre 1979 y 1999 , solamente el 1\% (16 medicamentos) fueron para enfermedades tropicales y tuberculosis(21). Mientras la I+D se dirige principalmente a enfermedades como la depresión, la obesidad o el colesterol, enfermedades como la tuberculosis, la diarrea o la malaria, responsables del $90 \%$ del problema sanitario mundial, reciben solo el $0,3 \%$ de los recursos destinados a investigación(22:74).

\section{Conclusión}

En el caso de Chile, la situación del acceso a los medicamentos es especialmente delicada. En comparación con otros países en desarrollo, como India, Argentina y Brasil, se encuentra en una posición de mayor vulnerabilidad frente al fortalecimiento mundial de los estándares de protección de las patentes farmacéuticas, cuya titularidad es controlada por empresas farmacéuticas internacionales. De partida, no introdujo instrumentos de política social o industrial capaz de desarrollar localmente un sector farmacéutico de innovación, y en 1991, en forma previa al Acuerdo ADPIC de la OMC, incorporó legislativamente la protección mediante patente a los medicamentos.

Con todo, el impacto del Acuerdo ADPIC para los países en desarrollo y países menos adelantados ha sido de envergadura en el ámbito farmacéutico. Antes de este acuerdo, más de 50 países —incluidos India, Argentina y Brasil- contemplaban en sus legislaciones la prohibición de patentes sobre productos farmacéuticos, lo cual obedecía precisamente a razones de salud pública, particularmente al temor de que las patentes limitaran el acceso a un bien esencial como es el medicamento. Con su entrada en vigencia han debido implementar la protección de patentes de medicamentos. Por ello, no puede extrańar los efectos perjudiciales que este acuerdo provoca en los países en desarrollo y países menos adelantados, manifestados en la Declaración de Doha relativa al Acuerdo sobre los ADPIC y la Salud Pública.

Además, el preocupante diagnóstico de la Declaración de Doha se agrava para numerosos países, entre los cuales se encuentra Chile, que han suscrito con potencias extranjeras TLC's con normas "ADPIC Plus", que refuerzan la protección de las patentes farmacéuticas y suelen limitar o anular la aplicación de las "flexibilidades" ADPIC. Luego de analizar en detalle este acuerdo comercial y sus implicancias para el acceso a los medicamentos, la Relatoría Especial de las Naciones Unidas ha exhortado a los países en desarrollo y los países menos adelantados a estar atentos a las actividades que pueden violar el derecho a la salud y de abstenerse de introducir en sus leyes nacionales y en tratados de libre comercio normas más estrictas que las contempladas en el Acuerdo ADPIC.

Dos comentarios finales. Primero, la importancia que adquiere para el acceso a los medicamentos básicos que los conflictos suscitados entre derechos de propiedad intelectual y la protección de la salud pública, por la aplicación e interpretación de las normas del Acuerdo ADPIC, se resuelvan claramente a favor de esta última. Partiendo por las controversias emblemáticas que enfrentan desde 2010 a India y Brasil contra la Unión Europea, y que generan inquietud en cuanto a la continuidad del suministro de medicamentos genéricos. Segundo, la necesidad de explorar nuevos marcos regulatorios de propiedad intelectual, en especial 
para las enfermedades desatendidas, las que al no generar un mercado atractivo y rentable para es- timular I+D, perpetúan la miseria y enfermedad para muchas personas alrededor del planeta.

\section{Referencias}

1. Grover A. Doc A/HRC/17/43. Informe del Relator Especial sobre el derecho de toda persona al disfrute del más alto nivel posible de salud fisica y mental. Ginebra: Naciones Unidas; 2011.

2. Hunt P. Doc E/CN.4/2004/49/Add.1. Informe del Relator Especial sobre el derecho de toda persona al disfrute del más alto nivel posible de salud física y mental: misión en la OMC. Nueva York: Naciones Unidas; 2004.

3. Allard R. El derecho humano a la atención primaria de salud en la lucha contra las enfermedades infecciosas. Tesis Doctoral, Universidad Complutense de Madrid, Facultad de Derecho, 2012. [Disponible en: http://eprints.ucm.es/15024/1/ T33707.pdf].

4. Grover A. Doc A/HRC/11/12. Informe del Relator Especial sobre el derecho de toda persona al disfrute del más alto nivel posible de salud física y mental. Ginebra: Naciones Unidas; 2009.

5. Hunt P. Doc A/63/263. Annex: Human Rights Guidelines for Pharmaceutical Companies in relation to Access to Medicines. In: Report of the Special Rapporteur on the right of everyone to the enjoyment of the highest attainable standard of physical and mental health. New York: United Nations; 2008.

6. Comisión de Derechos de Propiedad Intelectual, Innovación y Salud Pública. Salud pública, innovación y derechos de propiedad intelectual. Ginebra: OMS; 2006.

7. Comisión sobre Macroeconomía y Salud. Macroeconomía y salud: Invertir en salud en pro del desarrollo económico. Ginebra: OMS; 2002.

8. Hunt P. Doc E/CN.4/2006/48/Add.2. Informe del Relator Especial de las Naciones Unidas sobre el derecho al disfrute del más alto nivel posible de salud física y mental: Misión a Uganda. Nueva York: Naciones Unidas; 2006.

9. Deere C. The implementation Game: The TRIPS Agreement and the Global Politics of Intellectual Property Reform in Developing Countries. Oxford: Oxford University Press; 2008.

10. Sell S. Acuerdos de libre comercio con disposiciones ADPIC plus y acceso a medicamentos. En: Seuba X, (coordinador). Salud pública y patentes farmacéuticas: cuestiones de economía, politica y derecho. Barcelona: Bosch Editor; 2008.

11. Organización Mundial del Comercio. Entender la OMC. Quinta Edición. Ginebra: OMC, 2010: 55-60.

12. Allard R. El acceso a los medicamentos esenciales: revisión de las controversias ventiladas en la OMC por la aplicación del acuerdo sobre los ADPIC. En: Tórtora H, (coordinador). Las fuentes formales del derecho público. Santiago: Editorial Metropolitana; 2012.

13. Matsushita M, Schoenbaum T, Mavroidis P. The World Trade Organization, Law, Practice and Policy. Oxford: Oxford University Press; 2003: 419.

14. Gupta V. A mathematical Approach to Benefit-Detriment Analysis as a Solution to Compulsory Licensing of Pharmaceuticals under the TRIPS Agreement. Journal of International and Comparative Law 2005; 13: 631-660.

15. Castro R, Deik C. Salud pública, patentes de productos farmacéuticos y licencias obligatorias en el Acuerdo sobre los ADPIC: una mirada desde el tercer mundo. International Law, Revista Colombiana de Derecho Internacional 2008; 13: 165-214.

16. Schmitz C. Propiedad Intelectual a la luz de los Tratados de Libre Comercio. Santiago: LexisNexis; 2005: 191-238.

17. Bigorra J. Uso racional de los medicamentos. Aspectos éticos. En: Uso racional de los medicamentos. Aspectos éticos. Cuadernos de la Fundació Víctor Grífols i Lucas, No 8. Barcelona: Fundació Víctor Grífols i Lucas y Editorial Doyma; 2003: 9-18.

18. Camí J. Conflicto de intereses e investigación clínica. Medicina Clínica 1995; 105: 174-179.

19. Steinbrook R. Financial conflicts of interest and the NIH. New England Journal of Medicine 2004; 350(4): 327-330.

20. Páez R. La investigación de la industria farmacéutica: ¿̨condicionada por los intereses del mercado? Acta Bioethica 2011; 17(2): 237-246.

21. Trouiller P, Olliaro P, Torreele E, Orbinski J, Laing R, Ford N. Drug development for neglected diseases: a deficient market and a public-health policy failure. The Lancet 2002; 79(8): 2188-2189.

22. González B. Medicalización, Errores Médicos \& Industria Farmacéutica. 2a edición. Asturias: Althaea Editores; $2012: 71$.

Recibido: 3 de octubre de 2013

Aceptado: 17 de enero de 2014 